\title{
Politique
}

\section{Représentation politique et représentation statistique du peuple}

\section{Édouard Cloutier}

Numéro 9, hiver 1986

Démocratie et libéralisme

URI : https://id.erudit.org/iderudit/040514ar

DOI : https://doi.org/10.7202/040514ar

Aller au sommaire du numéro

Éditeur(s)

Société québécoise de science politique

ISSN

0711-608X (imprimé)

1918-6584 (numérique)

Découvrir la revue

Citer cet article

Cloutier, É. (1986). Représentation politique et représentation statistique du peuple. Politique, (9), 39-59. https://doi.org/10.7202/040514ar d'utilisation que vous pouvez consulter en ligne.

https://apropos.erudit.org/fr/usagers/politique-dutilisation/ 


\title{
Représentation politique et représentation statistique du peuple
}

\author{
Édouard Cloutier \\ Université de Montréal
}

Je vais ici examiner sommairement la façon dont la pratique des sondages a influencé la pratique de la démocratie politique dans les sociétés occidentales contemporaines. Pour ce faire, il importera d'abord de préciser ce qui est entendu par «démocratie politique», puis de saisir les grandes lignes de l'évolution de sa pratique avant et après l'avènement des sondages, de sorte que puisse être comparé le mouvement autonome de la démocratie politique avec son mouvement sous l'influence hypothétique des sondages. Cette comparaison s'effectuera en termes de changements survenus dans le système des communications politiques entre dirigeants et dirigés.

L'examen des rapports systématiques entre la pratique de la démocratie politique et celles des sondages sera par ailleurs exploratoire et sommaire en ce sens qu'elle vise davantage à proposer certaines hypothèses de réponses qu'à établir une explication globale et certaine de la rencontre des deux phénomènes. 


\section{La démocratie politique.}

Trois expressions courantes définissent globalement la démocratie politique: gouvernement du peuple, par le peuple, pour le peuple. La première réfere à la source de la souveraineté, la seconde à son exercice et la troisième à ses objectifs.

\section{Gouvernement du peuple}

Concernant la première, il convient de considérer comme démocratique tout régime politique qui désigne le peuple comme origine de l'autorité suprême plutôt qu'une divinité comme en théocratie, qu'une famille héréditaire comme en monarchie, qu'une classe héréditaire comme en aristocratie ou qu'un groupe de familles héréditaires comme en oligarchie. Le peuple peut être conçu soit, selon l'idée traditionnelle de la démocratie, comme l'ensemble des citoyens, soit, selon l'idée contemporaine de la démocratie, comme l'ensemble des individus réels. Dans le premier cas, propre à Rousseau ainsi qu'à la Déclaration des Droits de l'homme de 1789, le citoyen ne s'incarne pas dans un être de chair et de sang mais dans un pur esprit dont la conscience, modelée par l'éducation civique, n'entend que les enseignements de la raison et d'où sont exclues toutes les préférences subjectives. Par contre, les individus réels sont souverains en tant que personnes concrètement situées puisque la réunion de leurs volontés constitue l'autorité suprême, peu importe que ces volontés paraissent raisonnables ou non. Selon Burdeau (1956: 42), «l'idée contemporaine de démocratie ne retient comme maître du pouvoir que le peuple réel...(c'est-àdire) ...le groupe auquel la réunion des hommes situés donne son authenticité sociologique». Je ferai de même. La pratique des sondages étant un phénomène extrêmement contemporains, il convient de la juger à partir du concept le plus contemporain de l'origine de la démocratie. 
Notons tout de suite que ce concept exclut formellement que l'on rende la souveraineté du peuple conditionnelle soit au contenu de sa volonté, soit à ses attitudes et comportements à l'égard de la chose publique. On ne peut donc théoriquement exiger du citoyen, pour qu'il soit démocratique, comme le font par exemple Berelson, Lazarsfeld et McPhee (1954): 306), qu'il agisse selon des principes, qu'il réfere au bien commun plutôt qu'au sien propre, qu'il soit rationnel, intéressé, bien informé et enclin à la participation.

\section{Gouvernement par le peuple}

En ce qui concerne l'exercice de la souveraineté populaire, il convient pour en bien dégager le sens, de rappeler l'exemple classique des cités antiques grecques où les élections «tenaient une place tout à fait secondaire dans la théorie démocratique» (Duverger, 1962: 76). L'Ecclesia, assemblée générale du peuple, se réunissait très fréquemment pour prendre directement les décisions essentielles. Dans un tel contexte, le peuple n'avait nul besoin d'être représenté au niveau législatif puisqu'il légiférait lui-même. Quant aux fonctions exécutives, multipliées à l'extrême et de très courte durée, elles étaient souvent assignées par tirage au sort plutôt que par élection, le renouvellement de mandat étant généralement prohibé. De la sorte, existait dans la pratique une rotation certaine des fonctions exécutives. On assurerait ainsi, selon Duverger (Ibidem), l'identité des gouvernants et des gouvernés par l'égalité des chances qu'avaient les citoyens d'exercer ces fonctions, aucun ne voulant concéder à aucun autre le droit d'exercer, hors le consentement de l'ensemble, son autorité sur lui.

Cette pratique d'exercice continue et directe de l'autorité du peuple ne peut toutefois se concevoir que dans la mesure où le peuple est relativement peu nombreux et dispose du temps 
nécessaire aux fréquentes délibérations. Dans les démocraties antiques, on satisfaisait ces conditions en restreignant, de jure et de facto, le peuple aux seuls citoyens à l'exclusion des esclaves et des étrangers.

Il peut paraître paradoxal que la démocratie grecque antique demeure aujourd'hui encore un modèle classique de la démocratie alors même que, dans son esprit comme dans sa pratique, elle ne considérait comme "peuple» que celui composé d'un ensemble de personnes, les citoyens grecs, qu'on appellerait maintenant «aristocratie». C'est donc le mode d'exercice de la souveraineté de l'aristocratie grecque, fondé sur l'égalité de ses propres membres, qui nous permet de considérer encore aujourd'hui ce régime comme un modèle démocratique.

Dans les démocraties contemporaines, à l'inverse des démocraties antiques, l'exercice direct et contenu de l'autorité populaire est tenue pour impossible à cause de la grande taille et de la dispersion géographique du peuple. Si l'on exclut ces rares moments où il investit massivement la place publique pour désigner de nouveaux dirigeants ou pour dire la loi, le peuple n'exerce donc plus directement son autorité que de façon ponctuelle, à l'occasion d'une élection ou d'un référendum. Entre temps, l'exercice indirect de son autorité se trouve assuré par la représentation politique.

\section{La représentation politique}

L'acte de représentation politique du peuple a vu sa signification évoluer considérablement au cours de l'histoire moderne. Ainsi, l'idée traditionnelle de la démocratie, telle qu'elle s'est implantée au XIX $\mathrm{XI}^{\mathrm{e}}$ siècle, a engendré une représentation de la collectivité où «juridiquement le pouvoir du peuple est tout entier inclus dans l'organe représentatif» (Burdeau, 1956: 40). L'organe représentatif s'approprie alors la souveraineté du peuple. L'idée 
contemporaine de démocratie conçoit plutôt la représentation comme exprimant une volonté qui préexisterait à l'élection des représentants, ces derniers ne pouvant en conséquence agir qu'en vertu d'une délégation temporaire de la souveraineté populaire. Dans ce cas, "le pouvoir demeure en dehors de l'appareil gouvernemental. Il n'est pas capté par lui, il le domine et le conditionne...» (Burdeau, 1956: 44-5).

\section{Gouvernement pour le peuple}

Quant aux objectifs de la démocratie, j'éviterai d'y faire référence car poser comme critère de la démocratie qu'elle doive être exercée "pour» le peuple m'apparaît logiquement inutile, une fois admise l'idée contemporaine de la démocratie, à savoir l'exercice de l'autorité suprême par le peuple. Quand le peuple exerce sa souveraineté par élection ou référendum, on ne peut concevoir qu'il ne l'exerce pas "pour» lui qu'en concevant aussi une raison, une logique qui lui soit impérieusement supérieure, ce que la souveraineté du peuple interdit.

La souveraineté du peuple, comme règle de supériorité sur toute autre raison, constitue l'essence même de la démocratie politique. Cette règle, selon laquelle le peuple n'a d'autres juges de ses actions que lui-même, n'est pas fondée en logique mais posée en principe avec l'acceptation de l'idée de la démocratie. Il n'est en effet pas logiquement inconcevable que le peuple prenne des décisions qui s'avèrent, à leur application, détrimentale pour lui. Il est, par contre, logiquement inconcevable, une fois posée la souveraineté du peuple, d'admettre aussi quelqu'un, en dehors de lui ou en son sein, qui puisse lui imposer ses décisions.

Non seulement la souveraineté du peuple ne tient pas à son infaillibilité, mais sa faillibilité constitue une condition nécessaire à sa souveraineté: le peuple a, comme tout souverain, droit à 
l'erreur. Du point de vue strictement politique d'ailleurs, la démocratie ne requiert pas du peuple qu'il dise ni le vrai ni le réel mais qu'il gouverne, c'est-à-dire qu'il exerce son autorité. La démocratie politique ne constitue ni une règle de foi qui prétend établir la vérité, ni une règle de science qui prétend établir la réalité. Sa seule prétention est d'établir une autorité. Pour ce faire, il n'est nullement nécessaire que cette autorité soit infaillible.

Par ailleurs, la démocratie politique, telle qu'ici conçue, doit être dissociée de la démocratie sociale qui «vise à établir entre les individus une égalité de fait» (Burdeau, 1956: 68). Dans la mesure en effet où la démocratie sociale prétend dire ce qui est "pour» le peuple en assignant à la démocratie l'objectif de la promotion de l'égalité, elle suppose que les individus devraient rechercher cette égalité générale nécessaire à leur plein accomplissement. Or, notre conception de la démocratie n'assigne aucun objectif à l'autorité du peuple. Par conséquent, même dans les cas où le peuple userait de son autorité pour promouvoir l'inégalité ou toute entorse à quelconque autre objectif social, la démocratie politique ne serait en aucune façon niée.

On ne saurait non plus poser que le mode démocratique d'exercice de l'autorité fondé sur l'égalité de citoyens, tel que le concevaient les anciens Grecs, nécessite, pour éviter de n'être qu'une démocratie formelle, la construction d'une société entièrement démocratique, d'où seraient exclues toutes les inégalités (Ibidem). Du point de vue strictement politique qui est le nôtre, l'égalité des citoyens dans l'exercice de l'autorité populaire ne nécessite, ni comme condition nécessaire ni comme condition suffisante, l'égalité des citoyens à tout autre point de vue. Une telle égalité sociale doit être considérée comme un, parmi d'autres, des objectifs possiblement souhaitables de l'exercice de l'autorité populaire, sans plus. 


\section{L'exercice de l'autorité par le peuple réel}

C'est donc l'exercice de l'autorité par le peuple réel qui constituera notre seul critère de démocratie. En conséquence, notre intention sera d'examiner comment la pratique des sondages a influencé la pratique de l'exercice de l'autorité par le peuple.

Cet exercice on l'a vu, ne peut, dans les démocraties contemporaines, être à la fois direct et continu. Il est plutôt soit direct mais ponctuel, soit continu mais indirect. L'élection et le référendum incarnent le premier cas; l'exercice de l'autorité par des personnes élues le second.

Par une élection, le peuple exerce directement son autorité en nommant les personnes qui exerceront, pour une période donnée, l'autorité en son nom. Par un référendum, le peuple exerce directement son autorité en approuvant ou en désapprouvant un texte de loi fondamental. Ces deux exercices ponctuels et directs fondent l'exercice continu et indirect de l'autorité populaire: d'une part, l'élu agira en tenant compte à la fois du mandat sollicité lors de son élection et de l'obligation éventuelle d'avoir à renouveler son mandat, d'autre part le résultat d'un référendum balisera les comportements législatifs et exécutifs des élus. Puisque l'exercice continu et indirect trouve sa source dans les exercices ponctuels et directs, il convient de centrer notre attention sur ces derniers, et plus spécifiquement sur l'élection dans la mesure où elle se pratique, règle générale, de façon encore plus intensive et régulière que le référendum.

Les élections sont donc considérées ici comme un fil que le peuple maintient à la patte des élus tout au cours de leur mandat, lequel fil confere à l'exercice de l'autorité par les élus leur caractère d'exercice indirect de l'autorité par le peuple. 
La représentation statistique du peuple

Les sondages consistent essentiellement en des questionnaires auxquels sont appelés à répondre des représentants statistiques du peuple. Convenablement posées et traitées, les réponses des représentants permettent de tracer un portrait généralement fidèle ( 19 fois sur 20) mais quelque peu flou (une marge d'erreur de $3 \%$ à $10 \%$ selon la taille de l'échantillon), des pensées, comportements et états du peuple.

Dans une perspective électorale, les questions visent à établir d'une part les intentions de vote et, d'autre part, les facteurs explicatifs de ces intentions, tels la satisfaction à l'endroit du gouvernement sortant, l'évaluation respective des chefs, des candidats et des programmes ainsi que diverses caractéristiques de la pensée (opinions, attitudes, croyances, perceptions, orientations idéologiques, prévisions), des comportements (votes antérieurs) et des états (âge, sexe, occupation, revenus, scolarité, situation géographique, degré d'urbanisation) de l'électorat.

Pour saisir comment la disponibilité de ce portrait statistique a affecté l'exercice de l'autorité par le peuple, il convient d'examiner sommairement ce qui se passait avant son introduction qu'on peut situer vers les années ' 30 , moment où sont crées les premières entreprises privées de sondage (Stoetzel et Girard, 1973: 56-7) et les premiers organismes gouvernementaux et universitaires de recherche par sondage (Turner et Martin, 1984: 26-7) et où débute la pratique des consultants en sondage auprès des candidats et des élus (Blumenthal, 1982, 40) (Max, 1981: 72) (Sabato, 1981:70) (Stoetzel et Girard, 1973: 52).

Posons tout d'abord qu'en contexte électoral, toutes les instances impliquées cherchaient, alors aussi bien qu'aujourd'hui, à établir un portrait de l'électorat, les candidats pour mettre au point des stratégies permettant de convaincre les électeurs, les électeurs pour distribuer leurs votes de façon à en tirer un plus 
grand profit, c'est-à-dire à maximiser les chances d'élection des candidats préférés et/ou à minimiser les chances des candidats honnis. Si l'intérêt des candidats pour un tel portrait parait évident, on ne peut non plus douter de celui des électeurs, comme en témoignent la pratique assez ancienne (les premiers cas répertoriés datent de 1824) de publication de pseudo-votes («straw votes») par les media (Robinson, 1932) et l'importance qu'ont toujours accordée les journalistes à l'évaluation de la popularité des divers candidats. La recherche du portrait électoral va donc de pair avec la reconnaissance du principe de la souveraineté populaire et de son application pratique, l'élection au suffrage universel. Elle ne saurait en conséquence être imputable à l'apparition des sondages.

Cependant, le portrait dessiné par les partis et les média avant l'avènement des sondages différait sensiblement de celui qu'a éventuellement permis le sondage. Il était en effet constitué essentiellement d'informations souvent disparates, sinon contradictoires, partielles et partiales, librement interprétées par des personnes ressources (i.e. membres des partis, journalistes) au gré de leurs divers intérêts et selon des méthodologies très intuitives. De plus, le parachèvement de ce portrait pouvait nécessiter plusieurs mois de travail.

Le portrait statistique du peuple, par contre, établi par un personnel entraîné et politiquement neutre sur la base d'un échantillon représentatif à l'aide de méthodologies précises, s'avère plus rapide et systématiquement plus certain, bien que non dénué d'erreurs. Le portrait offre en outre plus de profondeur dans la mesure où il permet de saisir assez précisément comment de multiples facteurs se combinent pour expliquer l'intention de vote.

De part sa supériorité générale comme représentation du peuple, le portrait statistique a graduellement prévalu sur son prédécesseur, tant dans les partis que dans les média. 


\section{Représentation politique VS représentation statistique}

L'introduction de la représentation statistique dans la vie politique ne pouvait laisser indifférents les représentants politiques puisque se trouvait par là concurrencé leur privilège de parler au nom du peuple tout entier. Tous les parlements occidentaux se sont en effet penchés sur la question à quelques reprises et sous diverses formes au cours des vingt-cinq dernières années (Cloutier, 1982). C'est la diffusion des résultats des sondages électoraux par les média, plutôt que leur utilisation par les partis politiques, qui a surtout retenu l'attention. Bien que les délibérations parlementaires n'ont, règle générale, que rarement abouti à des décisions législatives (Rhome, 1984), elles ont souvent donné lieu à l'évocation d'arguments relatifs au caractère démocratique de la diffusion des sondages électoraux. Ainsi, il fut reproché à cette diffusion, non seulement d'induire le peuple en erreur, de diminuer le caractère solennel du moment électoral et d'affecter l'ardeur des militants des partis, mais surtout de transformer la démocratie parlementaire en démocratie directe, privant par là les partis politiques et les candidats de l'exclusivité du discours. C'est par contre au nom du principe démocratique du droit populaire à l'information que les propositions d'interdiction de diffusion ont généralement été repoussées. Quant aux propositions pour règlementer la qualité de la diffusion des sondages, on les a rejettées en évoquant surtout le principe de la libre concurrence entre média et entre entreprises de sondage comme garantie ultime de cette qualité (Cloutier, 1982: 8-18). Seule la France a jugé bon d'exiger légalement que la diffusion des sondages s'accompagne de certaines indications méthodologiques et du dépôt auprès d'un organisme public de divers documents pertinents (Antoine, 1981), (Crouzet, 1985) (Lazareff, 1983).

Les parlements se sont aussi penchés sur la question de l'accès aux résultats des sondages faits pour la branche exécutive du 
gouvernement. Les parlementaires ont évoqué leur droit, en tant que représentants élus du peuple chargés de légiférer au nom du peuple, d'avoir accès à tous les sondages gouvernementaux, sans que certains parlementaires (i.e. les dirigeants du parti au pouvoir) ne soient privilégiés au détriment des autres. D'autres parlementaires ont aussi soutenu que le conditionnement de l'opinion publique par la propagande fondée sur les sondages gouvernementaux constituait une perversion de la démocratie parlementaire dans la mesure où il revenait formellement aux parlementaires d'interpréter les volontés de la population sans qu'on ne les ait modifiées auparavant (Cloutier, 1983: 19).

Les représentants élus du peuple, surtout ceux de l'opposition parlementaire, ont donc craint que l'avènement des sondages ne réduise leur fonction de porte-parole officiel de la volonté populaire, une autre voix que la leur se faisant désormais entendre, aussi bien auprès des dirigeants que du peuple lui-même. Bien plus, les sondages permettaient que s'engagent des communications directes entre le peuple et les dirigeants au dessus et en dehors du canal formel de la représentation politique que constituent les parlements.

\section{Des rapports dirigeants-peuple sans représentation politique}

Les parlementaires craignent à juste titre d'être laissés à l'écart du rapport peuple-dirigeants car les sondages permettent aux dirigeants de communiquer avec le peuple sans passer par les parlementaires (Sabato, 1984: 187). Leur usage croissant s'inscrit dans le contexte, propre aux sociétés industrialisées, du déclin des communications sociales indirectes faites par voie de représentation, tels les partis politiques et les groupes de pression, au profit des communications directes rendues possibles par les mass media, les élections et les sondages (Antoine, 1979: 5). Certains 
auteurs attribuent à ce dernier type de communication la diminution des taux de participation électorale, la croissance de l'apathie à l'égard de la politique et le déclin des partis politiques (Blumenthal, 1982: 17-9) (Rubin, 1977: 144). Examinons ces trois présumées conséquences.

Il y a tout d'abord lieu de constater que si l'usage des sondages et des mass media s'est effectivement accompagné d'une décroissance de la participation électorale aux États-Unis, tel n'a généralement pas été le cas dans les pays d'Europe et au Canada. D'autre part, pour ce qui est de la Suisse, Burgat fait une constatation contraire à celle supposée ci-haut. C'est selon lui, le taux élevé d'abstentionnisme électoral qui explique, entre autres facteurs, le faible développement de la pratique des sondages en Suisse (1984: 29). Cet abstentionisme, en tant qu'indicateur du peu d'intérêt pour la politique en général et pour les sondages politiques en particulier, décourage la commandite de sondages par les medias. De plus, l'abstentionisme électoral s'accompagnerait de la non participation aux sondages, ce qui en augmenterait les coûts et partant en diminuerait la demande. On trouve donc des exemples de tous les rapports logiques entre la pratique des sondages et le déclin de la participation électorale: ils se produisent de pair, sans que l'on puisse dire lequel influence l'autre, et se produisent aussi indépendemment l'un de l'autre. Dans ces circonstances, on peut difficilement soutenir que la première cause le second.

Notons par ailleurs que le déclin de la participation électorale ne peut invalider l'hypothèse générale du rapport entre la croissance de communications directes et la décroissance des communications indirectes puisque l'élection au suffrage universel est considérée comme un exemple type de la communication directe et ne saurait, par conséquent, servir d'indicateur pour la communication indirecte. Le rapport négatif général tient au fait que l'information qui circule entre les dirigeants et le peuple n'est plus entièrement 
médiatisée par les partis et les groupes comme c'était le cas avant l'apparition des media de masses, puis des sondages.

Si l'on ne peut imputer à la pratique des sondages le déclin de la participation électorale, il semble par contre que cette pratique affecte certaines autres formes de participation politique. Ainsi, lors des dernières élections générales en Grèce, pays où la diffusion des sondages est interdite en période électorale et où leur confection rapide est rendue très difficile en raison des déficiences du réseau téléphonique, les foules partisanes qui se sont constituées de plus en plus nombreuses pendant la campagne électorale auraient rempli, dans une relation symbiotique avec les media, la fonction ailleurs impartie aux sondages de tracer le portrait du peuple à ses propres yeux. Ce phénomène rare d'une élection sans sondage mais avec media, permet de supposer que la présence des sondages électoraux entrave la constitution des foules partisanes.

De même, on peut considérer que les réponses que fournissent les représentants statistiques du peuple aux questions des sondages constituent aussi une forme de participation politique, moins exigeante certes que la participation active dans les partis et les groupes, mais tout de même non négligeable. Lang et Lang font état de recherche qui indiquent que les personnes appelées à répondre à des sondages sont susceptibles de voir leur intérêt pour la chose publique s'accroître, leur quête d'informations pertinentes s'intensifier, leurs opinions se clarifier et même leur participation électorale augmenter (1984). Bien que relativement marginal, cet effet participationiste des sondages augmente avec le nombre grandissant des sondages pratiqués et s'étend probablement, par un effet de halo, sur les personnes que fréquentent habituellement les représentants statistiques du peuple.

Pour ce qui est du déclin des partis politiques, il ne fait aucun doute que la perte de l'exclusivité de la fonction de canalisation des messages bi-directionnels entre les dirigeants et le peuple 
conduit à une atrophie partielle des partis, lesquels gardent cependant une partie de leurs fonctions antérieures: générer des candidatures et des programmes, mobiliser une force de travail bénévole à l'appui de leur présentation, fournir une image socialement négociée entre les représentants de diverses factions réellement constituées dans la société plutôt qu'une image, telle qu'en produisent les sondages, mathématiquement établie à partir des caractéristiques individuelles des représentants statistiques.

On ne peut conclure pour autant que les sondages, en contribuant à l'atrophie des partis, nuisent à l'exercice de l'autorité par le peuple. Il n'est en effet pas évident que la fonction de médiatisation des informations entre le peuple et les dirigeants ait toujours été adéquatement assumée par les partis et les groupes; on a vu plus haut que le portrait populaire qu'ils traçaient souffrait, de par la façon même dont il était constitué, de biais et d'imprécisions.

Il n'est enfin pas évident non plus que la participation politique, via les partis, les groupes et même les élections, soit logiquement un indicateur privilégié de l'exercice de l'autorité par le peuple. On peut en effet concevoir que, même quand toutes les possibilités de participer lui sont offertes, une partie du peuple choisisse de s'abstenir d'exercer son autorité pour toutes sortes de raisons: faible intérêt pour les enjeux, non pertinence des candidatures et des programmes, évaluation du peu d'effet anticipé d'une participation, etc. Dans certains cas, l'abstention est une forme d'exercice de l'autorité qui peut entraîner autant de conséquences espérées que la participation.

Des rapports médiatisés entre le peuple et les dirigeants

Les rapports de communication qu'établissent les sondages et les media entre le peuple et les dirigeants n'ont été qualifiés de directs qu'en ce sens qu'ils contournent les intermédiaires, 
dont les représentants officiels ou officieux du peuple. Doit-on pour autant les considérer comme des exercices directs de l'autorité par le peuple? Pour répondre à cette question, prenons, tel qu'entendu précédemment, l'Ecclésia grecque comme modèle de référence, c'est-à-dire un lieu physiquement restreint où non seulement la voix du peuple atteint, sans aucune espèce d'intermédiaire technique ou humain, l'oreille des dirigeants et, plus encore, un lieu où le peuple se parle à lui-même, simultanément en tant qu'ensemble dirigeant et en tant qu'ensemble dirigé, car rappelonsle, le peuple aristocrate grec légiférait lui-même et mandatait, pour de courtes périodes et le plus souvent par le hasard systématique (i.e. tirage au sort), un grand nombre de ses membres à exercer, sans capacité de reconduction, de multiples fonctions. Dans ces conditions, les rapports dirigés-dirigeants deviennent si diffus qu'on peut les concevoir comme à la limite de la disparition.

Par contre, les rapports d'autorité, tels qu'on les trouve dans les sociétés occidentales contemporaines, s'établissent dans deux lieux distincts, dont on peut difficilement soutenir qu'ils contribuent à l'exercice direct de l'autorité par le peuple. Les parlements constituent le premier lieu. Physiquement restreint, il permet un rapport sans intermédiaire non pas entre le peuple lui-même, mais entre personnes qui représentent le peuple parce qu'élues par lui. L'autre lieu est celui, physiquement étendu, des territoires couverts par les mass media et les sondages. Entre la voix du peuple qui parle aux dirigeants via les sondages et la voix que les sondages font entendre aux dirigeants, s'interposent de multiples médiations techniques (le questionnaire, le téléphone, la poste, l'ordinateur) et humaines (les interviewers, superviseurs, créateurs de questions et interprètes de réponses) qui traduisent le message populaire en le moulant chacune à sa façon. De même, entre la voix des dirigeants qui parle au peuple via les media et la voix que le peuple entend, les intermédiaires sont multiples dont en premier lieu les appareils de diffusion eux-mêmes qui jouissent 
d'une indépendance certaine vis-à-vis des dirigeants et qui sont techniquement contraints à des aménagements considérables des messages provenant des dirigeants.

Une médiation nombreuse et variée marque donc les rapports dirigeants-peuple dans les sociétés contemporaines. Nimmo et Combs soutiennent d'ailleurs que peu de gens ont une expérience directe de la politique et que, pour la grande majorité d'entre eux, les réalités politiques sont strictement médiatisées par les communications de masse et de groupe (Nimmo et Combs, 1983: XV). À l'inverse, on peut soutenir que des dirigeants ont assez rarement une connaissance directe du peuple, informée comme ils le sont surtout par des représentants officiels ou officieux des partis et des groupes, par les media et par les sondages, le tout transmis et structuré par des équipes de conseillers. Si les sondages ont ajouté une courroie de transmission sans représentation au flot des informations qui circulent du peuple vers les dirigeants, ils n'en demeurent pas moins eux-mêmes fortement médiatisés et les messages qu'ils produisent traduits par les interprètes que sont les conseillers.

\section{De la médiation partisane à la médiation non partisane}

Parmi ces conseillers, les consultants spécialisés en marketing politique ont eu tendance, particulièrement au cours de la dernière décennie, à occuper une place de plus en plus importante auprès des dirigeants, tout d'abord en période électorale, puis dans la gouverne quotidienne des États. Cette place, qu'ils ont progressivement ravie à des conseillers traditionnellement issus des partis et des groupes, les consultants la doivent à leur capacité d'user scientifiquement et artistiquement des sondages et des media beaucoup plus qu'à leur attachement partisan à l'égard des dirigeants (Blumenthal, 1982) (Perry, 1984) (Sabato, 1981) (Chagall, 1981). 
Du point de vue de l'exercice direct de l'autorité populaire, l'avènement en tant que tel de ces consultants change peu de chose. Bien sûr, ils constituent un groupe très restreint de personnes par rapport au peuple, n'ont pas été désignés par le peuple et agissent dans le secret, mais toutes ces caractéristiques sont également propres aux anciens "party boss» et à leurs descendants contemporains, les conseillers politiques spécialisés dans l'organisation des partis et des groupes. On a aussi exprimé l'avis que ces consultants en viendraient à supplanter, de facto sinon de jure, les dirigeants élus du peuple, tant leur influence grandit auprès de ces derniers (Rubin, 1977: 166). Rien n'indique pourtant qu'il en soit ainsi. La majorité des consultants restreignent leurs activités aux périodes électorales (Sabato, 1981: 40-3). Quant à ceux qui s'impliquent dans la gouverne, ils ont tôt fait de découvrir jusqu'à quel point leur influence, même croissante, doit être conciliée avec celle des conseillers plus traditionnels, lesquels ont conservé l'oreille attentive des dirigeants (Beal et Hinckley, 1984).

Il faut par ailleurs noter que si les consultants affichent une indépendance certaine par rapport aux partis, leur contribution n'en est pas moins politique pour autant, non seulement par le rôle qu'ils sont appelés à jouer dans les campagnes électorales et la gouverne des États, mais aussi parce que leur engagement auprès des candidats et des dirigeants s'avèrent très souvent politiquement motivé, certains choisissant de ne servir que des causes qu'ils perçoivent comme de gauche et de droite, selon les cas (Blumenthal, 1982) (Perry, 1984) (Sabato, 1981) (Chagall, 1981).

\section{L'autorité par les sondages}

Les sondages, on l'a vu, s'inscrivent avec les media dans le nouveau lieu des communications entre le peuple et les dirigeants. 
En tant que tels, ils ne peuvent manquer d'affecter l'exercice de l'autorité, objet même des rapports dirigeants-dirigés, puisqu'ils y interjectent un portrait du peuple dont les deux parties se doivent de tenir compte.

Nombreux sont en effet les exemples de résultats de sondages qui ont contribué à modifier des décisions prises par des dirigeants (Beal et Hinckley, 1984) (Turner et Martin, 1984: 51). Rideau prétend même, en s'appuyant sur sa pratique comme consultant auprès du président de la République française, que les sondages, plus précisément l'opinion à travers eux, nous gouvernent (1985 A: 193-5) ou, tout au moins, ont amené le Président à partager son pouvoir avec l'opinion publique (1985 B: 79). Par contre, on trouve aussi des exemples où les dirigeants ont agi à l'encontre de l'opinion nettement majoritaire, tel le cas, au Canada et en France, de l'abolition de la peine de mort.

Par ailleurs, Edwards soutient que si le Président américain a constamment besoin de l'appui du public pour gouverner avec efficacité, la lecture qu'il peut faire de l'opinion publique via les sondages, les résultats électoraux et le courrier qui lui est adressé, est approximative au mieux. Les sondages selon lui ne mesurent généralement pas l'intensité de l'opinion, ne sont pas suffisamment spécifiques relativement aux enjeux réels et ne surviennent pas toujours au moment opportun pour la prise de décision (Edwards, 1983: 2-3, 8-13). L'influence que le peuple exerce sur les dirigeants par la voie des sondages doit donc être considérée comme réelle, mais limitée, surtout si l'on tient compte du fait que les dirigeants cherchent eux aussi, comme ils l'ont toujours fait, à influencer le peuple.

D’après Bon (cité par Burgat, 1984: 33), l'inclusion des sondages dans la boucle des communications entre le peuple et les dirigeants a conduit à transformer cet instrument scientifique de connaissance et d'observation en arme politique et partisane 
dont la valeur d'argumentation l'emporte sur la part de vérité qu'elle contient. Il est indéniable que les sondages servent, entre autres outils, aux dirigeants pour faire de la propagande (Nimmo, 1970: 108-9), c'est-à-dire tenter de modifier les pensées, comportements et états du peuple à l'aide de la persuasion plutôt que de la force (Durandin, 1982: 11). Les sondages permettent aux dirigeants de mesurer l'appui dont ils bénéficient dans le peuple et les raisons de cet appui. Forts de cette information, ils tentent de l'augmenter par l'émission de messages dont ils mesurent les effets au moyen d'autres sondages... à l'aide desquels ils préparent de nouveaux messages... Rien ne garantit toutefois qu'ils y parviennent. Outre le fait que la propagande des dirigeants pourra se heurter à une contre-propagande venant d'autres dirigeants et à une action préventive de la part des media, la précision toute relative des sondages et, surtout, l'absence de tout ensemble codifié de lois formelles expliquant les mouvements de l'opinion interdisent en effet que l'on considère la connaissance des sondages comme un moyen qui assure aux dirigeants l'appui populaire.

Qu'ils s'en servent à cette fin ne saurait d'ailleurs leur être reproché du point de vue démocratique. La rhétorique, art de convaincre, n'a-t-elle pas fleuri précisément dans les démocraties grecques? En définitive, les sondages auront contribué, avec les mass media, à l'avènement d'une nouvelle rhétorique sociale qui englobe, en la transformant, celle précédemment établie par les partis et les groupes.

L'Ecclésia médiatisée demeurera cependant toujours un lieu où le peuple n'exercera son autorité que par la représentation, fut-elle aussi statistiquement exacte que possible. Et, quand bien même cette représentation devenait très perfectionnée, elle ne saurait tenir lieu de l'autorité que le peuple exerce directement par son vote, à la fois parce qu'un recensement complet des voix qui veulent se faire entendre vaudra toujours mieux que le meilleur 
des sondages et, surtout, parce qu'un sondage sans vote ne saurait constituer un exercice, même indirect, d'autorité par le peuple.

\section{Références}

Jacques ANTOINE, "Le rôle de la Commission des sondages lors de la campagne pour les élections présidentielles en France en 1981 ", Revue française de marketing, no. 3, 1981, pp. 93-6.

Jacques ANTOINE, "La communication entre les administrations et leurs publics», texte présenté au colloque L'administration publique: perspectives d'avenir, Québec, mai 1979.

Richar S. BEAL and Ronald H. HINCKLEY, «Presidential Decision Making and Opinion Pools", The Annals of the American Academy of Political and Social Sciences, March 1984, pp. 72-84.

Bernard A. BERELSON, Paul F. LAZARSFELD et William N. Mc PHEE, Voting, Chicago: University of Chicago Press, 1954.

Sidney BLUMENTHAL, The Permanent Campaign, N.Y.: Touchstone Books, 1982.

Georges BURDEAU, La démocratie, Paris: Seuil, (Points), 1956.

François BURGAT, "Les sondages d'opinion: outil de marketing politique», Revue économique et sociale, Lausanne, janvier 1984.

Édouard CLOUTIER, «Les sondages en tant qu'objets de pouvoir», communication présentée au congrès de l'association canadienne de science politique, Vancouver, juin 1982.

Édouard CLOUTIER, "How Contemparary Governments Regulate their Own Surveys: Power Struggle over Survey Information», communication présentée au congrès annuel de l'association canadienne de science politique, Guelph, juin 1983.

Édouard CLOUTIER, «Entre l'État moderne et les masses: le sondage et le marketing», essai présenté au congrès mondial de l'Association internationale de science politique, Paris, juillet 1985.

David CHAGALL, The New Kingmakers. M.Y.: Harcourt Brace Jovanovich, 1981.

Philippe CROUZET, "La jurisprudence de la Commission de sondages, 1978-1984", Pouvoirs, no. 33, 1985, pp. 57-77.

Guy DURANDIN, Les mensonges en propagande et en publicité, Paris: PUF, 1982.

Maurice DUVERGER, Institutions politiques et droit constitutionnel, Paris: PUF, 1962.

George C. EDW ARDS III, The Public Presidency, N.Y.: St-Martin's Press, 1983.

Kurt LANG et Gladys ENGEL LANG, "The Impact of Polls on Public Opinions", The Annals of the American academy of Political and Social Science, March 1984, pp. 129133.

Alexandre LAZAREFF, Le droit des sondages politiqnes, Paris: LGDJ, 1983.

Alfred MAX, La république de sondage, Paris: Gallimard, 1981.

Dan NIMMO, The Political Persuaders, the techniques of Modern Election Campaigns. Englewood Cliffs: Prentice-Hall, 1970. 
Dan NIMMO et James E. COMBS, Mediated Political Realities, N.Y.: Longman, 1983. Roland PERRY, Elections sur ordinateurs, Paris: Laffont, 1984.

Bernard RIDEAU, L'illusion du pouvoir, Paris: La table ronde, 1985(a).

Bernard RIDEAU, "Présidentialisme et sondocratie: le modèle Giscard», dans Pouvoirs, no. 33,1985 (B), pp. 79-87.

Nils RHOME, «Report of the Ad hoc Commitee on National Restrictions on the Product and Release of Public Opinion Polls", WAPOR Newletter, no. 83, Summer 1984, pp. 14-5.

Bernard RUBIN, Media, Politics and Democracy, N.Y.: Oxford University Press, 1977.

Larry J. SABATO, The Rise of Political Consultants, N.Y.: Basic Books, 1981.

Larry J. SABATO, "New Campaign Techniques and the American Party System", dans le recueil de textes préparé par Vernon Bogdanor, Parties and Democracy in Britain and America, N.Y.: Praeger, 1984.

J. STOETZEL et A. GIRARD, Les sondages d'opinion publique, Paris: PUF, 1973.

Charles A. TURNER and Elizabeth MARTIN (ed.), Surveying Subjectives Phenomena, (2 volumes), N.Y.: Russel Sage Foundation, 1984.

Je tiens à remercier les personnes qui ont participé aux séminaires sur les «Théories et pratiques de la démocratie" et sur la "Socio-politique des sondages et du marketing" donné à l'Université de Montréal pour leur fertile contribution à la présente argumentation, en particulier Pierre P. Tremblay qui m'a indiqué certaines références. Merci également aux anonymes évaluateurs d'une version antérieure de cet essai. La présente mouture leur doit de très nombreux éléments de clarification. 